Onkologie 1991;14:200-202

\title{
Inhalt, Vol. 14, No. 3, 1991
}

\section{Contents}

Ubersichtsarbeiten

Fortschritte beim kuratíven Einsatz der Chemotherapie bei Kopf-Halskarzinomen*

Volling, P., Schroder, M

1st Verapamil eine geeignete Substanz zur Überwindung der Zytostatikaresistenz?*

Harder, S

Hormonelle Behandlung des fortgeschrittenen Mammakarzinoms: Therapiesequenzen,

Substanzkombinationen, neue hormonell wirksame Medikamente

Worst, $\mathrm{P}$

212

218

Review Articles

Recent Advances of Curative Chemotherapeutic Management of Head and Neck Cancer* Volling, P., Schroder, M 205

Verapamil: A Promising Agent for Circumvention of Multidrug- Resistance? *

Harder, S 212

Hormonal Therapy of Advance Breast Cancer: Sequential Treatment, Combined Hormonal

Modalities, New Drugs

Worst, P 218

\section{Originalarbeiten}

Rationale und Stellenwert von Präoperativen Therapiemaßnahmen bei Patienten mit lokal fortgeschrittenem Mammakarzinom*

Jakesz, R.,Steger, G., Rainer,H 228

Prognosefaktoren von Patienten mit zerebralen Metastasen in der frühen postoperativen Phase*

Sachsenheimer, W. , Bimmler, T. , Piotrowski, W 234

Supportive Betreuung terminal krebskranker Patienten zu Hause und in einem Tages-Hospiz

Kleeberg, U.R., Kerekjarto, M., Kaden, H.,

Wagner-Bastemeyer, R., Kur, A., Lehmann, G., Schul·z, K.,

Bogan, G., Reichel, L., Erdmann, H 240

Antibiotische Behandlung der Sepsis mit Teicoplanin bei knochenmarktransplantierten

Patienten*

Fauser, A.A., Lang, E., Schmid, J.,Dölken, G., Kreisel, W.,

Löhr, G.W 248

Unterschiedliche CKII-Aktivitäten in menschlichen, kolorektalen Schleimhäuten, Adenomen und Karzinomen*

Pistorius, K., Seitz, G., Remberg, K., Issinger, O.G 256 
Steigerung der Zytotoxizität spezifischer monoklonaler Antikörper gegen humane Karzinomzellen durch GM-CSF in vitro

Staib,L.,Link, K.H., Büchler, M 261

Original Paper

The Rationale and Value of Preoperative Treatment Modalities in Patients with Locally

Advanced Breast Carcinoma*

Jakesz, R.,Steger, G., Rainer,H 228

Prognostic Factors of Patients with Cerebral Metastases in the Early Postoperative Phase*

Sachsenheimer, W., Bimmler, T., Piotrowski, W 234

Supportive Care of the Terminally Ill Cancer Patient at Home and in a Day-Hospice

Kleeberg, U.R., Kerekjarto, M., Kaden, H.,

Wagner-Bastemeyer, R., Kur, A., Lehmann, G., Schul·z, K.,

Bogan, G., Reichel, L.,Erdmann, H 240

Teicoplanin for the Treatment of Sepsis in Bone Marrow Transplant Recipients*

Fauser, A.A., Lang, E., Schmid, J., Dölken, G., Kreisel, W.,

Löhr, G.W 248

Differential CKII Activities in Human Colorectal Mucosa, Adenomas and Carcinomas*

Pistorius, K., Seitz, G., Remberg, K., Issinger, O.G 256

GM-CSF-Induced Increase in ADCC Against Human Cancer Cells in vitro

Staib,L.,Link, K.H.,Büchler, M 261

Fortsetzu $\prod g$ auf Seite 202

Continued on page 202

Internationale Zeitschrift fur Krebsforschung und -behandlung

Band 14, Heft 3, Juni 1991

Inhalt

Contents

Laufende klinische Studien

Hochmaligneperiphere-T-Zell-Lymphome: Therapie-Ergebnisse einer retrospektiven offenen multizentrischen Studie

Kuse, R., Lennert, K., Calavrezos, A., Tiemann, M.,

Engelhard, M 268

Erste Ergebnisse einer Induktionstherapie mit

fraktioniertem Aclacinomycin, Etoposid und

Cytosinarabinosid bei (myelo-)monozytärer Leukamie

in höherem Alter und/oder nach myelodysplastischem

Syndrom

Krieger, O., Kasparu, H., Weber, E., Lutz, D 272

Ongoing Clinical Trials

High-Malignant Peripheral T-Cell-Lymphomas: Treatment Results of a Retrospective Open

Multicenter Trial

Kuse, R. , Lennert, K. , Calavrezos, A. , Tiemann, M. ,

Engelhard, M

268 
First Results of an Induction Treatment with

Fractionated Aclacinomycin, Etoposid and

Intermediate-Dose Cytosinarabinosid in (Myelo-)

Monocytic Leukemia of Elderly Patients and/or After

Myelodysplastic Syndrome

Krieger, O., Kasparu, H.,Weber, E., Lutz, D 272

Kasuistiken

Erfolgreich durch Radiotherapie behandelte kutane Manifestationen des Morbus Waldenström

Latz, D., Mende, U

275

Casuistic Contributions

Successfully Treated Specific Cutaneous Manifestations of Morbus Waldenström with

Radiotherapy

Latz, D., Mende, U

275

Kongreßmitteilungen

Erfassung von Lebensqualität in der Onkologie -Konzepte, Methodik und Anwendung.

Heidelberg, 10.-12. Mai 1990

Flechtner, H 277

Mitteilungen onkologischer Gesellschaften

\begin{tabular}{lll} 
AIO-Mitteilungen & \multicolumn{2}{c}{. 278 } \\
Impressum & 199 & \\
Inhaltsverzeichnis & & 200 \\
Industrieforum & 289 & \\
Buchbesprechungen & & 290 \\
Hinweise für Autoren & & 291
\end{tabular}

* diese Arbeit erscheint in englischer Sprache

Congress Reports

Towards the Measurement of Quality of Life in Oncology - Concepts, Methods, and Application

-Heidelberg, May 10.-12.1990

Flechtner, H 277

Reports of Oncological Societies

AIO-Reports 278

Imprint

199

Contents 200

Industrial Forum 289

Book Reviews 290

Instructions to Authors

*this article is printed in English

Bibliographischer Hinweis: Inhaltsverzeichnisse dieser Zeitschrift erscheinen regelmäßig in current contents ${ }^{\circledR}$ sowie in anderen bibliographischen Diensten. 